\title{
NOVA TÉCNICA DE BLOQUEIO DO PLEXO BRAQUIAL EM CÃES
}

\author{
NEW TECHNIQUE OF BRACHIAL PLEXUS BLOCK IN DOGS
}

\begin{abstract}
Fábio Futema ${ }^{1}$ Denise Tabacchi Fantoni ${ }^{2}$ José Otávio Costa Auler Junior ${ }^{3}$ Silvia Renata Gaido Cortopassi ${ }^{4}$ Andrea Acaui $^{5}$ Angelo João Stopiglia ${ }^{6}$
\end{abstract}

\section{RESUMO}

O objetivo do presente estudo foi analisar a viabilidade e a eficácia de uma nova técnica para o bloqueio do plexo braquial em cães. Para tanto, foram utilizados 11 cães, machos $e$ fêmeas, idade e peso variáveis e mestiços. Os animais foram prétratados com acepromazina e a indução da anestesia foi realizada com propofol. Posteriormente, os animais foram submetidos ao bloqueio do plexo braquial que constou da associação da técnica de múltiplas injeções com o emprego do estimulador de nervos e a técnica da palpação arterial como ponto de referência para a localização dos nervos. Utilizou-se como anestésico local, a bupivacaína com vasoconstritor administrado na dose total de $4 \mathrm{mg} / \mathrm{kg}$ a $0,375 \%$. O volume total foi dividido em 4 partes iguais, administradas na velocidade de 30 segundos cada, com o objetivo de se atingir a maior quantidade de nervos. O tempo necessário para realização da técnica foi de 11,30 $\pm 4,54$ minutos; o período de latência para o bloqueio motor foi de 9,70 \pm 5,52 minutos $e$ para o bloqueio sensitivo foi de 26,20 \pm 8,86 min. , sendo a duração da analgesia de 11:00 \pm 0:45 horas. Em 90\% dos animais, o bloqueio foi efetivo, constatado através da anestesia de todo membro torácico distal à articulação escápulo-umeral. A única complicação observada foi a hipotensão arterial desenvolvida em um animal. Mediante os resultados obtidos, pode-se pressupor que as cirurgias envolvendo o membro torácico distal à articulação escápulo-umeral poderão ser utilizadas com auxílio desta nova técnica do bloqueio do plexo braquial, bem como na analgesia pós-operatória de longa duração.

Palavras - chave: plexo braquial, estimulador de nervos periféricos, cães, bupivacaína.

\section{SUMMARY}

The aim of this study was to evaluate the viability and efficacy of a new technique of brachial plexus block in dogs.
Eleven mongrel dogs of different ages and weight, both male and female were used. Animals were pre-medicated with acepromazine and induction of anesthesia was performed with propofol. The brachial plexus block was achieved with the combination of the following techniques: multiple injections, nerve stimulator and the axilary artery pulse to locate the nerves. Bupivacaine was the local anesthetic employed at a total dose of $4 \mathrm{mg} / \mathrm{kg}$. This dose was divided in 4, that were administered in 30 seconds each, to achieve more nerves as possible. The time spent to perform the whole procedure was $11.30 \pm 4.54$ minutes; onset time for motor and sensitive block were $9.70 \pm 5.52$ and $26.20 \pm$ 8.86 respectively. Duration of analgesia was $11.0 \pm 0.45$ hours. The block was effective in $90 \%$ of animals what was assessed through complete anesthesia of the front limb distally to the shoulder joint. Hypotension in one animal was the sole complication observed. With the results obtained we can suggest the use of this technique for front limb surgery performed distally to the shoulder joint as well as a pos-operative analgesic approach in dogs.

Key words: brachial plexus, peripheral nerve stimulators, dogs, bupivacaine.

\section{INTRODUÇÃO}

A anestesia regional é uma opção adequada para operações de membro superior em regime ambulatorial, ocupando um importante espaço na anestesia humana. $\mathrm{Na}$ anestesia veterinária, sua utilização é comumente empregada em grandes animais (BHOJANI et al., 1981). Em pequenos animais, a preferência pela anestesia geral ainda é a predominante. Dentre as inúmeras vantagens da anestesia regional, pode-se citar: redução do estresse \footnotetext{
Zeferino da Costa 82, apto 51,01526-060, São Paulo, SP. E-mail: fabio_futema@uol.com.br.

${ }^{2}$ Médico Veterinário, Professor Doutor, FMVZ, USP.

${ }^{3}$ Médico, Professor Titular, Faculdade de Medicina, USP

${ }^{4}$ Médico Veterinário, Professor Doutor, Faculdade de Medicina Veterinária, Universidade Paulista

${ }^{5}$ Médico Veterinário, Autônomo.

${ }^{6}$ Médico Veterinário, Professor Titular, FMVZ, USP.

${ }^{1}$ Médico Veterinário, Pós-graduando, Faculdade de Medicina Veterinária e Zootecnia (FMVZ), Universidade de São Paulo (USP). Rua 
cirúrgico (ANAND \& CARR, 1989), menor índice de mortalidade e morbidade quando comparados aos da anestesia geral (CHRISTOPHERSON et al., 1993), a necessidade de intubação orotraqueal é praticamente inexistente (URBAN \& URQUHART, 1994) e presença de analgesia pós-operatória (QUANDT \& RAWLINGS, 1996).

No que diz respeito à anestesia do plexo braquial, diversos autores descreveram diferentes técnicas com resultados nem sempre satisfatórios. Lindhorst, 1931,apud OLIVEIRA (1970) objetivou realizar a anestesia do membro torácico do cão através do bloqueio do plexo braquial, injetando o anestésico em diferentes quantidades e concentrações, diretamente no plexo braquial. Porém, os resultados obtidos foram insatisfatórios. Já TUFVESSON (1951) indicou a anestesia do plexo braquial em todas as intervenções cirúrgicas do membro torácico do cão realizadas distalmente à articulação do cotovelo. A sua técnica consistiu na utilização de $10 \mathrm{ml}$ de lidocaína, a $2 \%$, através da inserção da agulha na depressão triangular formada pela união da borda anterior do músculo supraespinhal com a parede do tórax e a borda dorsal do músculo braquicefálico. Sendo assim, a agulha foi inserida na direção caudal, entre a parede lateral do tórax e o músculo subescapular até a espinha da escápula.

NUTT (1962) utilizou o bloqueio do plexo braquial em casos clínicos. O bloqueio foi realizado segundo a técnica descrita por TUFVESSON (1951), mudando somente a concentração e o volume do anestésico local. Para tanto, o autor valeu-se de lidocaína, a 3\%, empregando um volume de 1 a $3 \mathrm{ml}$. Este autor indicou o bloqueio do plexo braquial nas intervenções cirúrgicas abaixo da articulação úmero-radio-ulnar. $\mathrm{O}$ que vem ao encontro com as observações de TUFVESSON (1951). No entanto, Vallat, 1959, apud, OLIVEIRA (1970) realizou o bloqueio do plexo braquial em cães, obtendo anestesia do membro torácico do cão, dista a articulação escápulo-umeral.

A utilização dos estimuladores de nervos para localização dos nervos a serem bloqueados foi descrita há mais de 30 anos por GREENBLATT \& DENSON (1962) e WRIGHT (1969). Segundo estes autores, esses estimuladores para localização dos nervos, aumentam significativamente o sucesso do bloqueio, sugerindo que esta técnica poderia ser extremamente útil em pacientes psicóticos, comatosos e torporosos, o que justificaria seu emprego na Medicina Veterinária (MOENS, 1995).

O estimular ideal é aquele que possui regulagem da amperagem, permitindo alterações freqüentes da mesma, durante a localização dos nervos.
Inicialmente, altas correntes são usadas para provocar as contrações musculares, e então, progressivamente se reduz a amperagem até o momento em que se consegue a contração com a menor amperagem, o que indica a proximidade da agulha com o nervo (BASHEIN $\boldsymbol{e t}$ al., 1984). Estes autores recomendam que se inicie com $1 \mathrm{~mA}$, reduzindo até conseguir contrações com 0,3 a $0,5 \mathrm{~mA}$; neste ponto, injeta-se $3 \mathrm{ml}$ do anestésico local que produzirá a abolição das contrações musculares, indicando assim a proximidade da agulha com o nervo a ser bloqueado (BARANOWSKI \& PITHER, 1990; KOSCIELNIAK-NIELSEN, et al., 1997).

BASHEIN et al. (1984) recomendam a utilização de agulhas descartáveis revestidas, exceto na ponta, por material plástico não condutor, concentrando a corrente elétrica, o que acarreta contrações musculares somente quando a ponta da agulha estiver próxima do nervo.

Desta forma, objetivou-se, através desta pesquisa, analisar a viabilidade e eficácia de uma nova técnica de bloqueio do plexo braquial no cão através da administração de bupivacaína com vasoconstritor na dose de $4 \mathrm{mg} / \mathrm{kg}$ a $0,375 \%$, como anestésico local e o emprego de uma combinação de técnicas, a saber: técnica de múltiplas injeções, associadas ao de estimulador de nervos periféricos e à palpação arterial como ponto de referência para a localização dos nervos ao seu redor.

\section{MATERIAL E MÉTODOS}

Foram utilizados 11 cães machos e fêmeas, idade e peso variáveis, mestiços provenientes do canil geral da Faculdade de Medicina Veterinária e Zootecnia da Universidade de São Paulo. Os animais foram submetidos a jejum alimentar de 12 horas e hídrico de quatro horas. Os animais receberam $0,1 \mathrm{mg} / \mathrm{kg}$ de acepromazina ${ }^{\mathrm{a}}$ como medicação préanestésica pela via intravenosa, na veia cefálica do membro torácico direito. Decorridos 10 minutos, esta mesma veia foi canulada com cateter ${ }^{\mathrm{b}}$ de calibre 20 G. Uma vez estabelecido o acesso venoso, realizou-se a indução anestésica consistindo na aplicação de $6 \mathrm{mg} / \mathrm{kg}$ de propofol $^{\mathrm{c}}$, permitindo assim, a realização da técnica do bloqueio do plexo braquial e a cateterização da artéria femural para a mensuração da pressão arterial.

A mensuração da pressão arterial foi realizada através de cateter, de calibre $20 \mathrm{G}$, introduzido na artéria femural direita e acoplado ao transdutor de pressão, que estava conectado ao monitor de pressão $^{\mathrm{d}}$. Após a instalação da pressão arterial, as pressões arteriais sistólica, média e diastólica foram mensuradas nos seguintes momentos: M0 - imedia- 
tamente, após a instalação da pressão arterial e M1 imediatamente, após a administração do volume total do anestésico local.

Para o bloqueio do plexo braquial, utilizou-se bupivacaína ${ }^{\mathrm{e}}$ com vasoconstritor a $0,5 \%$, diluído com água destilada até a obtenção da concentração, a 0,375\%. Desta diluição, utilizou-se a dose de $4 \mathrm{mg} / \mathrm{kg}$, em todos os animais no membro torácico esquerdo. O preparo para a realização da técnica constou de tricotomia na região do vazio torácico e da região axilar, e anti-sepsia com álcooliodo-álcool.

Os nervos foram localizados através do estimulador de nervo periféricos ${ }^{\mathrm{f}}$, inserindo a agulha ${ }^{\mathrm{g}}$ lateralmente à artéria axilar, na região do vazio torácico, uma vez que os nervos que compõem o plexo braquial, nesta região, estão ao redor da referida artéria. Para certificar-se da correta localização da artéria axilar na região do vazio torácico, empregou-se a técnica de obstrução do fluxo sangüíneo. Uma vez que uma leve pressão sobre a referida artéria acarretasse desaparecimento do pulso anteriormente palpável na artéria axilar, na região axilar, estaria confirmada a posição da artéria axilar na região do vazio torácico.

$\mathrm{O}$ estimulador de nervos periféricos foi empregado inicialmente com uma corrente de $1 \mathrm{~mA} \mathrm{e}$ $1 \mathrm{~Hz}$, diminuindo-se gradativamente até a obtenção de contrações com menos de $0,5 \mathrm{~mA}$. Neste momento, com a seringa contendo $4 \mathrm{mg} / \mathrm{kg}$ de bupivacaína, a $0,375 \%$, com vasoconstritor acoplada à agulha do estimulador de nervos, foi realizada aspiração, onde certificou-se que a mesma não estava no leito vascular. Após esta etapa, administrou-se 0,1 $0,2 \mathrm{ml}$ de bupivacaína, comprovando-se a proximidade da agulha com o nervo, pela inibição quase que imediata da contratibilidade muscular. Comprovada a proximidade da agulha, iniciou-se a administração do volume total divididosem 4 partes iguais e administradas na velocidade de 30 segundos cada aplicação, com o intuito de se atingir o maior número possível de nervos.

A partir da aplicação do volume total de bupivacaína, avaliou-se: a) tempo do desenvolvimento da técnica: período compreendido entre a palpação da artéria axilar até a administração do volume total do anestésico; b) tempo de instalação do bloqueio sensitivo ou tempo de latência sensitiva: período compreendido entre o término da administração do anestésico até a ausência da resposta dolorosa provocada pelo pinçamento da região interdigital e da pele, tanto na porção cranial do rádio quanto da pele na porção cranial e lateral do úmero. A resposta dolorosa foi comprovada através da atitude do animal (resposta comportamental), frente aos estímulos dolorosos. Esta resposta comportamental foi identificada através da presença de meneios de cabeça, vocalização, tentativa de mordedura e movimentos de outras partes do corpo; c) tempo de instalação do bloqueio motor ou tempo de latência motor: período compreendido entre o término da administração do anestésico até a ausência da resposta motora provocada pelo pinçamento da região interdigital e da pele, tanto na porção cranial do rádio, quanto da pele na porção cranial e lateral do úmero. A resposta motora foi comprovada através da flexão do membro após os pinçamentos; d) duração da analgesia: período compreendido entre a instalação do bloqueio sensitivo e a volta da sensibilidade do membro comprovada através do método de pinçamento, já ora referido.

Após 24 horas da instalação do bloqueio, os animais foram reavaliados para constatação de eventuais complicações. Esta reavaliação foi realizada através das mensurações de temperatura, frequiência cardíaca, frequiência respiratória, deambulação, hematomas axilares e paralisia do membro.

\section{RESULTADOS}

Os pesos, volume de bupivacaína empregado, tempos para a realização da técnica, período para instalação da latência motor, o período para instalação da latência sensitiva e a duração da analgesia dos animais estudados estão demonstrados na Tabela 1. Apenas um animal apresentou complicações. Após o término da administração do anestésico local, o animal apresentou quadro de hipotensão arterial aguda, necessitando de tratamento com efedrina, como demonstrado na Tabela 2. Porém, o bloqueio foi efetivo, pois o mesmo não respondeu às avaliações de sensibilidade motora e sensitiva. Outro animal, após uma hora de espera para a instalação do bloqueio, respondeu positivamente aos testes de função motora e sensitiva. Sendo assim, obteve-se no presente estudo índice de $90 \%$ de sucesso do bloqueio.

Não foi observado nenhum sinal de intoxicação pelo anestésico local, bem como qualquer distúrbio no membro bloqueado, 24 horas após a instalação do bloqueio. A área de anestesia promovida pelo bloqueio do plexo braquial foi toda porção distal à articulação escápulo-umeral.

\section{DISCUSSÃO}

A eficácia do bloqueio do plexo braquial em cães é, ainda hoje, fato limitante para a difusão da técnica. Lindhorst, 1931, apud, OLIVEIRA (1970), foi o primeiro a estudar a anestesia do plexo 
Tabela 1 - Valores individuais dos pesos (kg), volume de bupivacaína (ml), tempos de realização da técnica (minutos), período de latência motora e latência sensitiva (minutos) e duração do bloqueio (horas), médias e respectivos desvios-padrões.

\begin{tabular}{|c|c|c|c|c|c|c|}
\hline Animal & $\begin{array}{l}\text { Peso } \\
(\mathrm{kg})\end{array}$ & $\begin{array}{c}\text { Volume } \\
\text { Bupivacaína } \\
\text { (ml) }\end{array}$ & $\begin{array}{c}\text { Duração da } \\
\text { técnica }\end{array}$ & $\begin{array}{l}\text { Latência } \\
\text { motora }\end{array}$ & $\begin{array}{l}\text { Latência } \\
\text { sensitiva }\end{array}$ & $\begin{array}{c}\text { Duração } \\
\text { do } \\
\text { bloqueio }\end{array}$ \\
\hline 1 & 14,6 & 15,57 & 6,0 & 5,0 & 10 & $11: 15$ \\
\hline \multirow[t]{2}{*}{2} & 13,5 & 13,49 & 10,0 & 10 & 30 & $12: 10$ \\
\hline & 16,0 & 17,06 & 8,0 & 5,0 & 22 & $11: 30$ \\
\hline 4 & 15,0 & 16,00 & 7,0 & 22 & 40 & $11: 15$ \\
\hline 5 & 16,6 & 17,70 & 20 & 10 & 25 & $11: 40$ \\
\hline 6 & 16,6 & 17,70 & 7,0 & 10 & 40 & $10: 00$ \\
\hline 7 & 12,7 & 13,54 & 15,0 & 10 & 15 & $10: 45$ \\
\hline 8 & 11,7 & 12,48 & 10,0 & 15 & 35 & 11:00 \\
\hline 9 & 15,3 & 16,32 & 15,0 & 5,0 & 25 & $9: 50$ \\
\hline 10 & 15,8 & 16,85 & 15,0 & 5,0 & 20 & $11: 40$ \\
\hline Média & 14,80 & 15,67 & 11,30 & 9,70 & 26,20 & 11:00 \\
\hline DP & 1,99 & 1,75 & 4,54 & 5,52 & 8,86 & $0: 45$ \\
\hline
\end{tabular}

DP Desvio-Padrão.

Tabela 2 - Valores individuais das pressões arteriais $(\mathrm{mmHg})$ : sistólica (PAS), média (PAM) e diastólica (PAD), médias e respectivos desvios-padrões.

\begin{tabular}{|c|c|c|c|c|c|c|}
\hline \multirow[t]{2}{*}{$\begin{array}{c}\text { Animal } \\
\mathrm{N}=\end{array}$} & \multicolumn{2}{|c|}{$\begin{array}{c}\text { PAS } \\
(\mathrm{mmHg})\end{array}$} & \multicolumn{2}{|c|}{$\begin{array}{c}\text { PAM } \\
(\mathrm{mmHg})\end{array}$} & \multicolumn{2}{|c|}{$\begin{array}{c}\text { PAD } \\
(\mathrm{mmHg})\end{array}$} \\
\hline & M0 & M1 & M0 & M1 & M0 & M1 \\
\hline 1 & 130 & 120 & 95 & 87 & 80 & 76 \\
\hline 2 & 110 & 60 & 86 & 45 & 75 & 35 \\
\hline 3 & 140 & 134 & 90 & 100 & 78 & 81 \\
\hline 4 & 100 & 112 & 77 & 86 & 65 & 71 \\
\hline 5 & 134 & 136 & 107 & 105 & 91 & 87 \\
\hline 6 & 84 & 121 & 63 & 90 & 52 & 68 \\
\hline 7 & 125 & 119 & 103 & 100 & 93 & 90 \\
\hline 8 & 108 & 113 & 80 & 93 & 70 & 81 \\
\hline 9 & 120 & 118 & 84 & 84 & 64 & 66 \\
\hline 10 & 124 & 134 & 84 & 89 & 67 & 72 \\
\hline Média & 117,5 & 116,7 & 86,9 & 87,9 & 73,5 & 72,7 \\
\hline DP & 17,04 & 21,75 & 12,79 & 16,58 & 12,60 & 15,43 \\
\hline
\end{tabular}

DP Desvio-Padrão

braquial em cães. Mediante os resultados obtidos, este autor não recomenda a técnica na prática clínica. Neste trabalho, obteve-se sucesso em $90 \%$ dos animais submetidos à nova técnica de bloqueio do plexo braquial. O bloqueio do plexo braquial em cães, quando eficiente, é recomendado nas cirurgias distais à articulação úmerorádio-ulnar (TUFVESSON, 1951; NUTT, 1962). Contudo, na atual pesquisa, conseguiu-se a anestesia do membro torácico distal à articulação escápulo-umeral, o que está de acordo com as observações de Vallat, 1959, apud, OLIVEIRA (1970).

A técnica ora realizada baseou-se na técnica proposta por TUFVESSON (1951), associada às técnicas utilizadas na Medicina. Utilizou-se a técnica da palpação arterial como ponto de referência para a localização dos nervos ao seu redor, técnica esta, utilizada na abordagem axilar (GOLDBERG et al., 1987; PIPPA \& RUCCI, 1994), o emprego do estimulador de nervos para localização dos nervos proposto por GREENBLATT \& DENSON (1962) e WRIGHT (1969), e a técnica de múltiplas injeções (URBAN \& URQUHART, 1994; KOSCIELNIAK-NIELSEN $\boldsymbol{e} t$ al., 1997).

Em relação ao período de latência e duração do bloqueio, não foi possível uma análise comparativa com as técnicas preconizadas na Medicina Veterinária, pois, no presente estudo, o anestésico local, dose, concentração e velocidade de administração foram modificados. A bupivacaína tem sido o anestésico local de escolha para os procedimentos cirúrgicos de duração longa e na analgesia pósoperatória, por apresentar duração de longa ação e distinção entre anestesia sensitiva e bloqueio motor (SARMA, 1990; HICKEY et al., 1991; BRANDL \& TAEGER, 1991; HICKEY et al., 1992 ;DORMAN et al., 1994; VAINIONPÄÄ et al., 1995; PARIKH et al., 1995; RUCCI et al., 1995).

A utilização de vasoconstritor na bupivacaína tem o intuito de prolongar a duração da anestesia, diminuir a toxidade, o sangramento, ajudar na avaliação dose-teste e diminuir a toxidade sistêmica por reduzir a velocidade de absorção, minimizando assim a concentração sangüínea do anestésico (SWEITZER, 1997). Na presente pesquisa nenhum sinal de toxidade foi verificado.

A dose de bupivacaína empregada foi de $4 \mathrm{mg} / \mathrm{kg}$, valendo-se do estudo realizado por PIHLAJAMÄKI (1991), que correlacionou os níveis séricos máximos da concentração de bupivacaína no sangue (Cmax) com o peso corpóreo em pacientes 
submetidos ao bloqueio do plexo braquial. Em todos os pacientes utilizou-se $200 \mathrm{mg}$ de bupivacaína independentemente dos pesos. Desta forma, encontrou-se doses diferentes em seus pacientes que variaram de $1,92 \mathrm{mg} / \mathrm{kg}$ a $5,10 \mathrm{mg} / \mathrm{kg}$, não observando nenhum sinal de toxidade, mesmo naqueles em que foi utilizada a dose máxima. QUANDT \& RAWLINGS (1996) recomendam a dose de 1,5 a $2 \mathrm{mg} / \mathrm{kg}$ de bupivacaína para o bloqueio do plexo braquial em cães. MUIR \& HUBBELL (1995) recomendam $2 \mathrm{mg} / \mathrm{kg}$ de bupivacaína como dose máxima permitida. Contudo, FELDMAN et al. (1989), estudando a toxidade da bupivacaína em cães, obtiveram $4,3 \mathrm{mg} / \mathrm{kg}$ como dose convulsivante. Segundo MUNSON et al. (1975) e MALAGODI et al. (1977), a dose necessária para induzir toxidade no sistema cardiovascular é de duas a quatro vezes mais do que a necessária para induzir a toxidade no sistema nervoso central. De acordo com os resultados obtidos por METS et al. (1992), a dose necessária para induzir parada cardiovascular em ratos foi de $12,3 \mathrm{mg} / \mathrm{kg}$. Contudo, no presente estudo, não foi observado qualquer sinal de toxidade.

De acordo com WINNE et al. (1979), no bloqueio do plexo braquial, o volume da solução injetada é fator relevante para o sucesso da anestesia. Na maioria da literatura médica, a dose do anestésico local para o bloqueio de plexo braquial está associada principalmente ao volume e concentração do fármaco escolhido. Nesta pesquisa, o volume médio de bupivacaína utilizado foi de $15,67 \mathrm{ml}$ em animais com pesos médios de $14,80 \mathrm{~kg}$. PIPPA \& RUCCI (1994) utilizaram em pacientes com peso entre 50 a $80 \mathrm{~kg}$ a combinação de lidocaína, $2 \%$, com bupivacaína, $0,5 \%$, na dose de $40 \mathrm{ml}$, sendo $20 \mathrm{ml}$ de cada fármaco no bloqueio do plexo braquial. VAINIONPÄÄ et al. (1995) utilizaram bupivacaína, $0,5 \%$, no bloqueio do plexo axilar; a dose do anestésico local foi de acordo com o peso do paciente: até $70 \mathrm{~kg}, 30 \mathrm{ml}$, entre 70 a $80 \mathrm{~kg}$, a dose foi de $35 \mathrm{ml} \mathrm{e}$, acima de $80 \mathrm{~kg}$, utilizaram-se $40 \mathrm{ml}$

No que diz respeito especificamente à bupivacaína, diferentes concentrações têm sido utilizadas para diversos procedimentos no homem (HICKEY et al., 1991; HICKEY et al., 1992; BRANDL \& TAEGER, 1991; PARIKH et al., 1995). HICKEY et al. (1992) avaliaram em estudo comparativo a bupivacaína, $0,25 \%$, e a ropivacaína, $0,25 \%$, observaram necessidade de suplementação da anestesia com anestésico geral em 9 pacientes de 22 , estudados no grupo da bupivacaína e 8 pacientes de 22, estudados no grupo da ropivacaína. Mediante os resultados obtidos, os autores não recomendam a utilização de $0,25 \%$ de bupivacaína ou $0,25 \%$ de ropivacaína no bloqueio do plexo braquial. Contudo,
BLANDL \& TAEGER (1991) utilizaram bupivacaína a $0,375 \%$, obtendo concentração e volume adequados para o sucesso do bloqueio. No presente estudo, utilizou-se a bupivacaína a $0,375 \%$ com vasoconstritor, na dose de $4 \mathrm{mg} / \mathrm{kg}$.

Em relação ao estimulador de nervos periféricos, na atual pesquisa, estipulou-se $0,5 \mathrm{~mA}$ como corrente máxima para a administração do anestésico. Segundo o trabalho realizado na medicina por EIFERT et $\boldsymbol{a l} .$, (1994) a máxima corrente para a injeção do anestésico local deve ser inferior a $0,5 \mathrm{~mA}$, pois seus resultados demonstraram que as falhas do bloqueio ocorridas durante o estudo foram devidas à utilização de $0,6 \mathrm{~mA}$ para a administração do anestésico, enquanto os sucessos decorreram pela utilização de $0,5 \mathrm{~mA}$ como ponto de referência para a administração do anestésico, uma vez que quanto menor a miliamperagem, maior a proximidade da agulha ao nervo. Segundo RODRIGUEZ et al.(1995), a eficiência das técnicas regionais é diretamente proporcional à possibilidade de deposição da solução anestésica o mais próximo possível do elemento que se deseja bloquear.

A técnica de múltiplas injeções realizada no homem permite que mais de um nervo seja localizado, levando a um índice maior de efetividade do bloqueio, pois, segundo BARANOWSKI \& PITHER (1990), quanto maior a quantidade de nervos localizados durante o bloqueio do plexo braquial mais efetivo será o bloqueio. Sendo assim, foi proposto neste estudo a utilização da técnica de múltiplas injeções não somente para melhorar a eficácia do bloqueio, mas também para diminuir o tempo de latência, assim como sugerido por KOSCIELNIAKNIELSEN et al. (1997).

A difusão do anestésico local sobre o plexo braquial no homem é fator fundamental para o sucesso do bloqueio (RUCCI et al., 1995). Frente a estas observações, utilizou-se no presente estudo a velocidade de administração de 30 segundos, para cada aplicação, sendo a dose do anestésico local dividida em quatro partes iguais. Assim, a técnica requereu dois minutos para a administração de todo o anestésico local. RUCCI et al. (1995) sugeriram melhora na difusão do anestésico e eficácia do bloqueio, empregando esta mesma velocidade de administração.

Durante o bloqueio nervoso periférico, que envolve uma série de nervos como no bloqueio do plexo braquial, o bloqueio motor, com freqüencia, desenvolve-se previamente ao sensitivo (CARPENTER \& MACKEY, 1996). Estas observações coincidem com os achados no atual trabalho, onde a latência motora e sensitiva foram 9,7 e 26,2 minutos respectivamente. 
O tempo necessário para a realização da técnica adicionado à latência do bloqueio acarreta um tempo prolongado para que o paciente esteja apto para o início da cirurgia. No presente estudo, este tempo foi em média 11,30 minutos. De forma semelhante, KOSCIELNIAK-NIELSEN et al. (1997) obtiveram em média 9,5 minutos para a realização da técnica do bloqueio do plexo braquial na medicina. Neste estudo, o período de duração da analgesia foi em média de 11 horas, o que conferiu analgesia pós-operatória de longa duração, como observado na medicina por SARMA (1990); BRANDL \& TAEGER (1991); DORMAN et al. (1994); INBERG et al. (1995).

RODRIGUEZ et al. (1996) estudaram 40 pacientes submetidos ao bloqueio do plexo axilar, utilizando mepivacaína, 1,4\%, associado a bicarbonato de sódio a $8,4 \%$. Em dois pacientes, verificouse inexplicada hipotensão após a administração do anestésico. Estes pacientes foram tratados com efedrina. Semelhante ao ocorrido com RODRIGUEZ et al. (1996), no presente estudo um animal apresentou queda da pressão arterial, necessitando também de tratamento com efedrina.

Após 24 horas do bloqueio, não foi verificado nenhum comprometimento do membro bloqueado, o que vem ao encontro com os resultados obtidos por DORMAN $\boldsymbol{e} \boldsymbol{t} \boldsymbol{a l}$. (1994) e INBERG $\boldsymbol{e} \boldsymbol{t} \boldsymbol{a l}$. (1995).

Frente aos resultados obtidos, pode-se concluir que o bloqueio do plexo braquial é técnica factível de ser empregada no cão, promovendo anestesia de toda a porção distal à articulação escápulo-umeral, o que permite sua utilização nas cirurgias de membro torácico.

\section{FONTES DE AQUISIÇÃO}

${ }^{a}$ Acepran a 0,2\% - Univet S.A.-Indústria Veterinária

${ }^{\mathrm{b}}$ Abbocath T Plus - Produtos Hospitalares Abbott

${ }^{c}$ Diprivan a $1 \%$ - Zêneca Farmacêutica do Brasil Ltda.

${ }^{\mathrm{d}}$ Biomonitor 7 - Bese Engenharia de Sistemas

${ }^{\mathrm{e}}$ Marcaína a 0,5\% - Astra Química e Farmacêutica Ltda.

${ }^{\mathrm{f}}$ Stimuplex DIG - Bbraun Melsungen AG

${ }^{\mathrm{g}}$ Agulha A 100 0,8 x 100 mm - Bbraun Melsungen AG

\section{REFERÊNCIAS BIBLIOGRÁFICAS}

ANAND, K.J.S., CARR, D.B., The neuroanatomy, neurophisyology, and neurochemistry of pain, stress, and analgesia in newborns and children. Pediatric Clinics of North America, v. 36, n. 4, p. 795-822, 1989.

BARANOWSKI, A.P., PITHER, C.E. A comparison of three methods of axilary brachial plexus anaesthesia. Anaesthesia, v. 45 , p. $362-365,1990$

BASHEIN, G., HASCHKE, R.H., READY, L.B. Electrical nerve location: Numerical and electrophoretic comparison of insulated vs unimsulated needles. Anesthesia and Analgesia, v. 63, p. 919,1984
BHOJANI, S.A., PARSANIA, R.R., VYAS, K.N., et al. A new approach for the brachial plexus block in cattle and buffalo. Indian Veterinary Journal, v. 58, p. 777-779, 1981.

BRANDL, F., TAEGER, K. The combination of general anesthesia and interscalene block in shoulder surgery. Anaesthesist, v. 40, n. 10, p. 537-542, 1991.

CARPENTER, R.L., MACKEY, D.C. Local anesthetic. In: BARASH, P.G., CULLEN, B.F., STOELTING, R.K. Clinical anesthesia. 3. ed. Philadelphia: Lippincott-Raven, 1996. Cap. 17, p. 413-439.

CHRISTOPHERSON, R., BEATTIE, C., FRANK, S., et al. Perioperative morbidith in patients randomized to epidural or general anesthesia for lower extremity vascular surgery. Anesthesiology, v. 79, p. 422-434, 1993

DORMAN, B. H., CONROY, J.M., DUC, T.A., et al. Postoperative analgesia after major shoulder surgery wirh interscalene brachial plexus blockade: etidocaine versus bupivacaine. Southern Medical Journal, v. 87, n. 4, p. 502$505,1994$.

EIFERT, B., HAHNEL, J., KUSTERMANN, J. Axillary blockade of brachial plexus. A prospective study of blockade sucess using eletric nerve stimulation. Anaesthesist, v. 43, n. 12, p. 780-785, 1994.

FELDMAN, H.S., ARTHUR, G.R., COVINO B.G. Comparative systemic toxicity on convulsant and supraconvulsant doses of intravenous ropivacaine, bupivacaine, and lidocaine in the conscious dog. Anesthesia and Analgesia, v. 69, p. 794-801, 1989.

GREENBLATT, G. M., DENSON, J. S. Needle nerve stimulatorlocator: nerve blocs with a new instrument for locating nerves. Anesthesia and Analgesia, v. 41, n. 5, p. 599-602, 1962.

GOLDBERG, M. E., GREGG, C., LARIJANI, G.E., et al. A comparison of three methods of axillary approach to brachial plexus blockade for upper extremity surgery. Anesthesiology, v. 66, p. $814-816,1987$.

HICKEY, R., HOFFMAN, J., RAMAMURTHY, S. A comparasion of ropivacaine $0.5 \%$ and bupivacaine $0.5 \%$ for brachial plexus block. Anesthesiology, v. 74, p. 639-642, 1991.

HICKEY, R., ROWLEY, C.L., CANDIDO, K.D., et al. A comparative study of $0.25 \%$ ropivacaine and $0.25 \%$ bupivacaine for brachial plexus block. Anesthesia and Analgesia, v. 75, p. 602-606, 1992.

INBERG, P., KASSILA, M., VILHHI, S., et al. Anaesthesia for microvascular surgery in children. Acta Anaesthesiologica Scandinavica, v. 39, p. 518-522, 1995.

KOSCIELNIAK-NIELSEN, Z.J., STENS-PEDERSEN, H.L., LIPPERT, F.K. Readiness for surgery after axillary block: single or multiple injection techniques. European Journal of Anaesthesiology, v. 14, p. 164-171, 1997.

MALAGODI, M.H., MUNSON, E.S., EMBRO, M.J. Relation of etidocaine and bupivacaine toxicity to rate of infusion in rhesus monkeys. British Journal of Anaesthesia, v. 49, p. $121-125,1977$.

METS, B., JANICKI, P.K., JAMES, M.F., et al. Lidocaine and bupivacaine cardiorespiratory toxicity is additive: a study in rats. Anesthesia and Analgesia, v. 75, p. 611-614, 1992. 
MOENS, Y. Brachial plexus block in goats using a nerve stimulator. Journal Veterinary Anaesthesia, v. 22, p. 39, 1995.

MUIR, W.W., HUBBELL, J.A.E. Veterinary Anesthesia. St. Louis: Mosby , 1995. Local anesthesia in dogs and cats: p. 96112.

MUNSON, E.S., TUCKER, W.K., AUSINSCH, B., et al. Etidocaine, bupivacaine, and lidocaine seizure thresholds in monkeys. Anesthesiology, v. 42, p. 471-478, 1975.

NUTT, P. Brachial plexus analgesia in dog. The Veterinary Record, v. 74, n. 32, p. 874-876, 1962.

OLIVEIRA, H.P. Contribuição para a anestesia do membro torácico do cão. Belo Horizonte -MG. 47 p. Tese (Mestrado em Medicina Veterinária) - Curso de Pós-graduação em Medicina Veterinária. Universidade Federal de Minas Gerais, 1970

PARIKH, R.K., RYMASZEWSKI, L.R., SCOTT, N.B Prolonged postoperative analgesia for arthrolysis of the elbow joint. British Journal of Anaesthesia, v. 74, p. 469-471, 1995.

PIHLAJAMÄKI, K.K., Inverse correlation between the peak venous serum concentration of bupivacaine and the weight of the patient during interscalene brachial plexus block. British Journal of Anaesthesia, v. 67, p. 621-622, 1991.

PIPPA, P., RUCCI, F.S. Preferential channelling of anaesthesic solution injectede within the perivascular axillary sheath. European Journal of Anaesthesiology, v. 11, p. 391-396, 1994.

QUANDT, J. E., RAWLINGS, C. R. Reducing postoperative pain for dogs: local anesthesic and analgesic techniques. Compendium on Continuing Education for Practicing Veterinrian, v. 18, n. 2, p. 101-111, 1996.
RODRIGUEZ, J., CARCELlER, J., BARCENA, M. Axillary brachial plexus anesthesia: electrical versus cold salina stimulation. Anesthesia and Analgesia, v. 83, p. 752-754, 1996

RODRIGUEZ, J., CARCELLER, J., BARCENA, M., et al. Cold saline is more effective than room temperature saline in inducing paresthesia during axillary block. Anesthesia and Analgesia, v. 81, p. 329-331, 1995.

RUCCI, F.S., PIPPA, P., BOCCACCINI, A., et al. Effect of injection speed on anaesthetic spread during axillary block using the orthogonal two-needle technique. European Journal of Anaesthesiology, v. 12, p. 505-511, 1995.

SARMA, V.J. Long-term continuous axillary plexus blockade using $0.25 \%$ bupivacaine. Acta Anaesthesiologica Scandinavica, v. 34, p. 511-513, 1990.

SWEITZER, B.J. Anestésicos locais. In: DAVISON, J.K., ECKHARDT III, W.F., PERESE, D.A. Manual de Anestesiologia Clínica. 4. ed. Rio de Janeiro: Medsi, 1997. Cap. 15, p. 172-179.

TUFVESSON, G. Anestesi av plexus brachialis. Nordisk Veterinaer Medicin, v. 3, p. $183-193,1951$.

URBAN, M.K., URQUHART, B. Evaluation of brachial plexus anesthesia for upper extremity surgery. Regional Anesthesia, v. 19, n. 3, p.175-182, 1994.

VAINIONPÄÄ, V.A., HAAVISTO, E.T., HUHA, T.M., et al. A clinical and pharmacokinetic comparison of ropivacaine and bupivacaine in axillary plexus block. Anesthesia and Analgesia, v. 81, p. 534-538, 1995.

WINNE, A.P., RADONJIC, R., AKKINENI, S.R., et al. Factors influencing distribution of local anesthetic injected into the brachial plexus sheath. Anesthesia and Analgesia, v. 58, p. 225-234, 1979.

WRIGHT, B.D. A new use for the block-aid monitor. Anesthesiology, v. 30, n. 2, p. 236-237, 1969.

Ciência Rural, v. 29, n. 1, 1999. 